\title{
Incident cerebral microbleeds and hypertension defined by the 2017 ACC/AHA Guidelines
}

\author{
Yiwei Xia ${ }^{1 \#}$, Yi Wang ${ }^{1 \#}$, Lumeng Yang ${ }^{1}$, Yiqing Wang ${ }^{1}$, Xiaoniu Liang ${ }^{2}$, Qianhua Zhao ${ }^{2}$, Jianjun Wu ${ }^{1,3}$, \\ Shuguang Chu ${ }^{4}$, Zonghui Liang ${ }^{5}$, Hansheng Ding ${ }^{6}$, Ding Ding ${ }^{2}$, Xin Cheng ${ }^{1}$, Qiang Dong ${ }^{1}$ \\ ${ }^{1}$ Department of Neurology, National Clinical Research Centre for Aging and Medicine, Huashan Hospital, State Key Laboratory of Medical \\ Neurobiology, Fudan University, Shanghai, China; ${ }^{2}$ Institute of Neurology, National Clinical Research Centre for Aging and Medicine, Huashan \\ Hospital, Fudan University, Shanghai, China; ${ }^{3}$ Department of Neurology, Jing'an District Center Hospital, Shanghai, China; ${ }^{4}$ Department of \\ Radiology, Shanghai East Hospital, Tongji University School of Medicine, Shanghai, China; ${ }^{5}$ Department of Radiology, Jing'an District Center \\ Hospital, Shanghai, China; 'Shanghai Health Development Research Center (Shanghai Medical Information Center), Shanghai, China \\ Contributions: (I) Conception and design: Y Wang, X Cheng, Q Dong; (II) Administrative support: X Cheng, Q Dong; (III) Provision of study \\ materials or patients: J Wu, S Chu, Z Liang, H Ding, D Ding; (IV) Collection and assembly of data: Y Xia, Y Wang, L Yang, Y Wang; (V) Data \\ analysis and interpretation: Y Xia, X Liang, Q Zhao; (VI) Manuscript writing: All authors; (VII) Final approval of manuscript: All authors. \\ "These authors contributed equally to this work. \\ Correspondence to: Xin Cheng, MD, PhD. Department of Neurology, Huashan Hospital Fudan University, 12 Wulumuqi Zhong Road, Shanghai, \\ 200040, China. Email: chengxin@fudan.edu.cn; Qiang Dong, MD, PhD. Department of Neurology, Huashan Hospital Fudan University, 12 \\ Wulumuqi Zhong Road, Shanghai 200040, China. Email: qiang_dong163@163.com.
}

Background: The cut-off for hypertension was lowered to blood pressure (BP) over 130/80 $\mathrm{mmHg}$ in the 2017 American College of Cardiology/American Heart Association (ACC/AHA) guideline. Whether the new definition of hypertension remains a potent risk factor of cerebral microbleeds (CMBs) is uncertain. We aimed to analyze the relationship between the new definition of hypertension and incident CMBs in a 7-year longitudinal community study.

Methods: This study is a sub-study of the Shanghai Aging Study (SAS). A total of 317 participants without stroke or dementia were included at baseline (2009-2011), and were invited to repeated clinical examinations and cerebral magnetic resonance imaging (MRI) at follow-up (2016-2018). CMBs at baseline and followup were evaluated on T2*-weighted gradient recalled echo (GRE) and susceptibility-weighted angiography (SWAN) sequence of MRI. We classified baseline BP into four categories: normal BP, elevated systolic BP, stage 1 hypertension and stage 2 hypertension according to the ACC/AHA guideline. We assessed the associations between BP categories and incident CMBs by generalized linear models.

Results: A total of 159 participants (median age, 67 years) completed follow-up examinations with a mean interval of 6.9 years. Both stage 1 and stage 2 hypertension at baseline were significantly related with a higher risk of incident CMBs (IRR 2.77, 95\% CI, 1.11-6.91, P=0.028; IRR 3.04, 95\% CI, 1.29-7.16, P=0.011, respectively), indicating dose-response effects across $\mathrm{BP}$ categories. Participants with $\geq 5$ incident $\mathrm{CMBs}$ or incident CMBs in the deep locations all had baseline stage 1 and 2 hypertension.

Conclusions: Participants with baseline stage 1 and stage 2 hypertension had a significantly higher risk of incident CMBs in this 7-year longitudinal community cohort.

Keywords: Cerebral microbleeds (CMBs); blood pressure (BP); ACC/AHA guidelines; longitudinal studies; cerebral small vessel disease

Submitted Jul 05, 2020. Accepted for publication Nov 15, 2020.

doi: 10.21037/atm-20-5142

View this article at: http://dx.doi.org/10.21037/atm-20-5142

^ ORCID: 0000-0002-8184-1228. 


\section{Introduction}

Cerebral microbleeds (CMBs), an imaging marker of cerebral small vessel diseases, are visualized as small rounded hypointense lesions on blood-sensitive magnetic resonance imaging (MRI) sequences (1), and are associated with recurrent stroke, dementia and mortality (2-4).

Cross-sectional $(5,6)$ and longitudinal studies $(7,8)$ revealed that blood pressure (BP) over $140 / 90 \mathrm{mmHg}$ was a crucial risk factor of CMBs in the general population. Since the Systolic Blood Pressure Intervention Trial (SPRINT) showed that aggressive control of BP resulted in lower rates of major cardiovascular events and mortality (9), cut-off for hypertension was lowered from $140 / 90$ to $130 / 80 \mathrm{mmHg}$ in the 2017 American College of Cardiology/American Heart Association (ACC/AHA) guideline (10). While several randomized clinical trials supported a target of $\mathrm{BP}$ lower than $130 / 80 \mathrm{mmHg}$ in secondary stroke prevention $(11,12)$, one study pointed out that there would be an increase in serious adverse events if the SPRINT intensive systolic blood pressure (SBP) treatment goal was fully implemented (13). The clinical value of this new definition of hypertension remains controversial (14-16).

Few studies have examined the significance of this new definition of hypertension in the prevalence and progression of CMBs. One cross-sectional study demonstrated that stage 1 hypertension (SBP: 130-139 mmHg, DBP: $80-89 \mathrm{mmHg}$ ) was independently associated with the prevalence of CMBs in a healthy population (17). However, to our knowledge, the relationship between baseline stage 1 hypertension and incident CMBs in longitudinal studies remains unclear.

In our study, we aimed to investigate the relationship between the new definition of hypertension and incident CMBs in a 7-year longitudinal community cohort study.

We present the following article in accordance with the STROBE reporting checklist (available at http://dx.doi. org/10.21037/atm-20-5142).

\section{Methods}

\section{Study population}

This study is a sub-study of the Shanghai Aging Study (SAS), which was a cohort study on the elderly community residents in Jing'an Temple, an urban community in Shanghai, China. We have published the detailed study protocol previously (18). A total of 350 adults over 60 years of age without dementia or stroke underwent baseline clinical examinations and cerebral MRI including T2*weighted gradient recall echo (GRE) sequence from 2009 to 2011. Of the 350 MRI examinations, 33 were excluded because of the missing of GRE sequence. Therefore, 317 participants were considered as baseline participants for this study. Exclusion criteria were listed as follows: (I) more than $50 \%$ stenosis of cerebral large vessels; (II) brain tumors or hydrocephalus; (III) MRI contraindications; (IV) incorporative or inability to complete the examination. All eligible participants were invited to repeated clinical examinations and cerebral MRI at follow-up (2016-2018), as described in our previous study (19).

\section{MRI}

From 2009 to 2011 at baseline, all eligible participants took cerebral MRI scans on a 1.5-Tesla GE scanner. The sequences were as follows: $\mathrm{T} 2{ }^{*}$-weighted gradient recalled echo (GRE), T1-weighed, T2-weighed, axial fluidattenuated inversion recovery (FLAIR), and magnetic resonance angiogram (MRA) sequence. We have described full acquisition details previously (20).

From 2016 to 2018 at follow-up, all eligible participants underwent repeated MRI scans on a 3.0-Tesla GE scanner with following sequences: Susceptibility Weighted ANgiography (SWAN) (slice thickness $2.0 \mathrm{~mm}$, flip angle 15 degree, TR/TE minimum/45 ms), 3D T1 BRAVO (slice thickness $1.2 \mathrm{~mm}$, flip angle 12 degrees), T2 PROPELLER (slice thickness $6.0 \mathrm{~mm}$, slice spacing $2.0 \mathrm{~mm}$, flip angle 140 degree, $\mathrm{TR} / \mathrm{TE}=7.586 \mathrm{~s} / 93.76 \mathrm{~ms}$ ), Cor CUBE FLAIR (slice thickness $2.0 \mathrm{~mm}$, TR/TE 6,000/90 ms), and MRA (slice thickness $1.4 \mathrm{~mm}$, flip angle 20 degree, TR/TE minimum/minimum).

CMBs were defined on $\mathrm{T} 2{ }^{*}$-weighted GRE at baseline or SWAN at follow-up as rounded areas of signal void with associated blooming, 2 to $10 \mathrm{~mm}$ in diameter (1). Signal voids were excluded in case of sulcal vessels, choroid plexus and pineal calcifications, symmetrical calcifications in the basal ganglia, and signal averaging from bone (21). Two neurologists with certification and registration assessed the number and location of CMBs at baseline and followup according to the Microbleed Anatomical Rating Scale (MARS) without knowing the clinical data of the participants (22). CMBs were assessed with good interrater reliability $(\mathrm{k}=0.75)$ and excellent intra-rater reliability $(\mathrm{k}=0.90)$. Results were confirmed by a senior neurologist if any disagreements exist. The participants were categorized into two groups according to CMBs location (i.e., strictly 
deep CMBs vs. lobar or infratentorial CMBs [with or without additional deep $\mathrm{CMBs}]$ ). Incident $\mathrm{CMBs}$ were defined as any new CMBs from baseline.

\section{Clinical assessment}

We evaluated baseline demographics, vascular risk factors and medication use, including age, sex, education, body mass index, current smoking, apolipoprotein $\mathrm{E}$ (APOE) genotypes, history of hypertension, diabetes mellitus, hyperlipidemia, cardiogenic disease, and use of antihypertensive, antidiabetic, lipid lowering, and antiplatelet or anticoagulation medication. Cardiogenic disease was defined as atrial fibrillation or coronary artery disease. APOE genotypes were assessed via DNA samples from blood or saliva (20). The presence of at least one $\varepsilon 4$ (or $\varepsilon 2$ ) allele was treated as being APOE $\varepsilon 4$ (or $\varepsilon 2$ ) positive. History of hypertension was defined by a self-reported medical history of hypertension, and was confirmed in medical records ( $\mathrm{SBP} \geq 140$ or $\mathrm{DBP} \geq 90 \mathrm{mmHg}$ ). Details of clinical assessment were previously published elsewhere (20).

After the participants rested on a comfortable ordinary chair for at least 5 minutes, baseline BP was measured by using a mercury sphygmomanometer with a cuff of appropriate size, following standard recommended procedures (23). Two readings of SBP and DBP were taken at 5-minute intervals, and SBP and DBP data were obtained by averaging each 2 readings (17). According to the 2017 ACC/AHA hypertension guidelines (10), participants' BP at baseline were categorized into 4 groups: (I) normal BP, SBP $<120 \mathrm{mmHg}$ and $\mathrm{DBP}<80 \mathrm{mmHg}$; (II) elevated systolic BP, SBP of 120 to $129 \mathrm{mmHg}$ and DBP $<80 \mathrm{mmHg}$; (III) stage 1 hypertension, SBP of 130 to $139 \mathrm{mmHg}$ or DBP of 80 to $89 \mathrm{mmHg}$; and (IV) stage 2 hypertension, $\mathrm{SBP} \geq 140 \mathrm{mmHg}$ or DBP $\geq 90 \mathrm{mmHg}$. Mean arterial pressure (MAP) was estimated using a formula in which the DBP was doubled and added to the SBP and that composite sum then was divided by 3 to estimate MAP. Pulse pressure (PP) was the SBP minus the DBP.

\section{Statistical analysis}

We performed statistical analysis on Stata v14.0 (StataCorp, College Station, Texas, USA). Graphs were drawn using Prism v7.0 (GraphPad Software, San Diego, CA). Median and interquartile range (IQR) were used to describe continuous variables because of the nonnormal distribution. Categorical variables were described using number and percentage. Continuous variables were compared using Mann-Whitney U test or Kruskal-Wallis test. Categorical variables were compared using Chi-square test or Fisher's exact test.

The associations between incident CMBs and baseline risk factors including BP categories were evaluated using univariable and multivariable generalized linear models (Poisson regression). Covariables were selected from univariable analyses with $\mathrm{P}<0.05$ or with known potential clinical significance $(7,17,24,25)$. These covariables were adjusted in multivariable models. All the subgroups of BP categories were put together in the models by using dummy variable categories. Normal BP was considered as the reference category.

All incident rate ratios, $95 \%$ confidence intervals and $\mathrm{P}$ values were estimated in a two-tailed fashion. $\mathrm{P}<0.05$ was considered statistically significant.

\section{Ethic statements}

The authors are accountable for all aspects of the work in ensuring that questions related to the accuracy or integrity of any part of the work are appropriately investigated and resolved. This study was approved by the medical ethics committee of Huashan Hospital, Fudan University, Shanghai, China (ID: 2016-359), and conducted in accordance with Chinese Good Clinical Practice of Pharmaceutical Products and the Declaration of Helsinki (as revised in 2013). Written informed consent was obtained from all participants or their legally acceptable representative.

\section{Results}

Of the 317 subjects, 28 people were deceased, 60 refused to be followed, 32 could not be reached, 11 moved to other communities, 9 could not participate actively due to dementia and tumor, and 18 had MRI contraindications. As a result, we were able to prospectively study 159 subjects with a mean interval of 6.9 years (Figure 1).

Table S1 showed the characteristics of the 159 participants at baseline and follow-up. Baseline median age was 67 years, and $44.7 \%$ were male. Mean follow-up interval was $6.9 \pm 0.4$ years (5.7 to 8.0 years). Compared to those followed participants, those lost to follow-up were significantly older with more baseline vascular risk factors and prevalence of microbleeds (Table S2).

The overall prevalence of CMBs at baseline and follow- 
up were $16(10.1 \%)$ and $53(33.3 \%)$ respectively. The number and location of CMBs at baseline and follow-up in the four BP groups were shown in Table S3. The prevalence of any microbleeds at baseline was higher in those with stage 1 hypertension (11.4\%) and stage 2 hypertension $(11.4 \%)$ than those with normal BP (4.8\%). A similar trend was observed in the prevalence of any microbleeds at follow-up (normal BP: 19.0\%, elevated systolic BP: $26.7 \%$, stage 1 hypertension: $31.4 \%$, stage 2 hypertension: $38.6 \%$ ).

Forty-six (28.9\%) participants developed incident CMBs during the follow-up period. The percentages of participants with incident $\mathrm{CMBs}$ in the four $\mathrm{BP}$ groups were shown in Figure 2. Participants with $\geq 5$ incident CMBs all

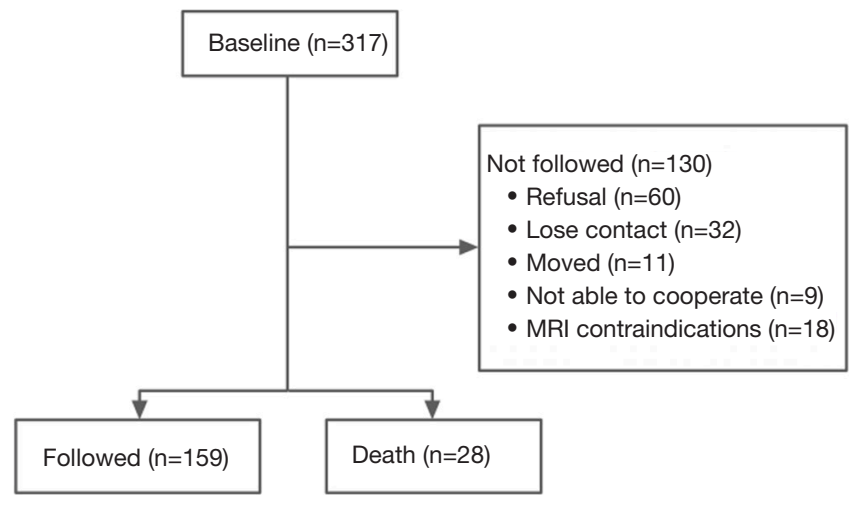

Figure 1 Flowchart of subject recruitment in Jing'an Temple Community. had baseline stage 1 and 2 hypertension (Figure $2 A$ ). With respect to CMBs location (Figure $2 B$ ), participants with incident strictly deep CMBs all had baseline stage 1 and 2 hypertension. No difference was found in the distribution of incident lobar or infratentorial CMBs.

The relationships between baseline predictors and incident CMBs were demonstrated in Table 1. In the univariable model, baseline stage 1 hypertension and stage 2 hypertension were significantly related with a higher incidence rate of CMBs (IRR 2.7, 95\% CI, 1.116.54, $\mathrm{P}=0.028$; IRR 3.22, 95\% CI, 1.41-7.38, $\mathrm{P}=0.006$, respectively). After multivariable adjustment, baseline stage 1 hypertension and stage 2 hypertension were also significantly associated with a higher incidence rate of CMBs (IRR 2.77, 95\% CI, 1.11-6.91, $\mathrm{P}=0.028$; IRR 3.04, 95\% CI, 1.29-7.16, $\mathrm{P}=0.011$, respectively), showing doseresponse effects across $\mathrm{BP}$ categories.

Participants with incident $\mathrm{CMBs}$ had significantly higher baseline SBP than those without incident $\mathrm{CMBs}(\mathrm{P}=0.024$, Figure 3). Participants who developed incident CMBs had a trend toward higher baseline DBP, MAP and PP; this association almost reached statistical significance for $\mathrm{PP}$ $(\mathrm{P}=0.057)$.

\section{Discussion}

In this 7-year longitudinal relatively healthy community cohort study, we found that $28.9 \%$ of participants developed incident CMBs. Participants with baseline stage 1 and stage
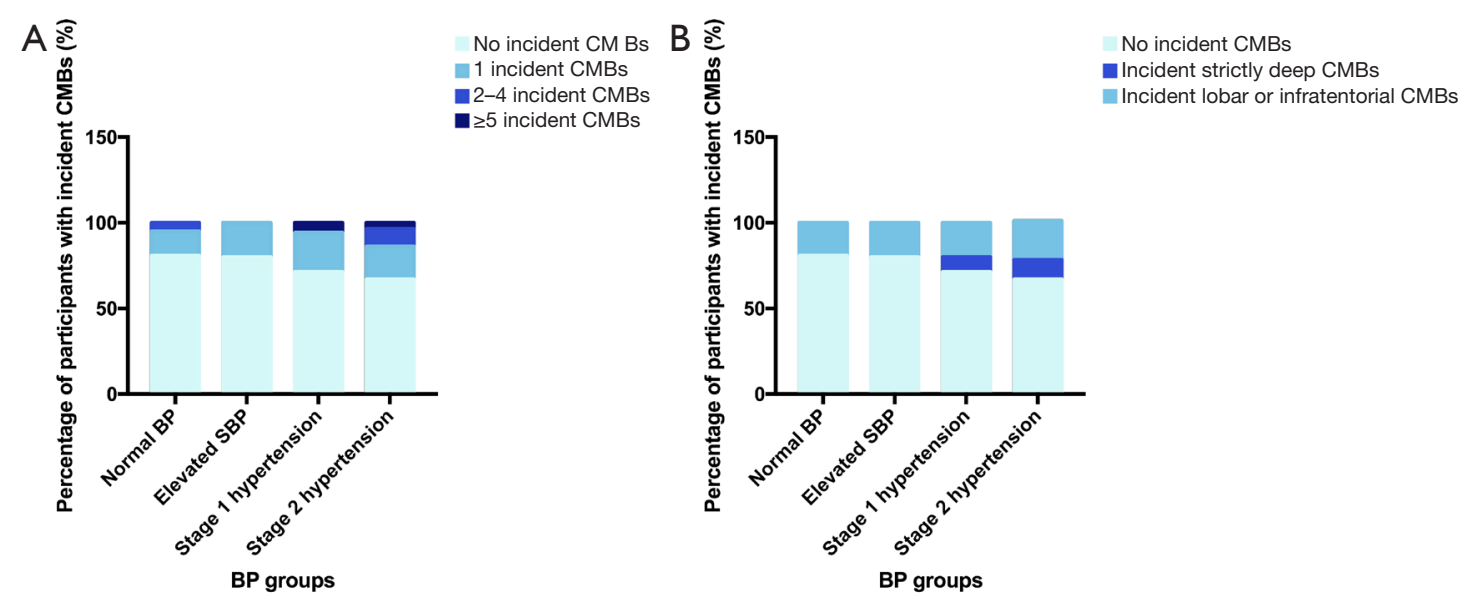

Figure 2 Percentage of participants with incident CMBs in each BP group. All participants (n=159) were categorized into four groups by baseline BP levels. Each column represents the distribution of participants with incident CMBs in each BP group. (A) Columns were assigned different colors in terms of number of incident CMBs; (B) columns were assigned different colors in terms of location of incident CMBs. CMBs, cerebral microbleeds; BP, blood pressure; SBP, systolic blood pressure. 
Table 1 Univariable and multivariable analyses between possible predictors and the incidence of cerebral microbleeds

\begin{tabular}{|c|c|c|c|c|c|c|}
\hline Possible predictors & \multicolumn{3}{|c|}{ Univariable } & \multicolumn{3}{|c|}{ Multivariable } \\
\hline Age & 1.07 & $1.03,1.10$ & $<0.001$ & 1.11 & $1.07,1.15$ & $<0.001$ \\
\hline Sex & 0.37 & $0.24,0.57$ & $<0.001$ & 0.17 & $0.10,0.29$ & $<0.001$ \\
\hline Body mass index & 1.04 & $0.99,1.09$ & 0.101 & 1.04 & $0.99,1.10$ & 0.140 \\
\hline ApoE $\varepsilon 4$ carriers & 1.20 & $0.74,1.94$ & 0.467 & 1.70 & $1.00,2.87$ & 0.049 \\
\hline ApoE $\varepsilon 2$ carriers & 0.63 & $0.35,1.11$ & 0.112 & 0.57 & $0.31,1.03$ & 0.063 \\
\hline \multicolumn{7}{|l|}{ BP categories } \\
\hline Normal BP & Ref & Ref & Ref & Ref & Ref & Ref \\
\hline Diabetes & 2.54 & $1.68,3.85$ & $<0.001$ & 2.75 & $1.76,4.31$ & $<0.001$ \\
\hline Hyperlipidemia & 0.46 & $0.30,0.71$ & $<0.001$ & 0.33 & $0.21,0.54$ & $<0.001$ \\
\hline Cardiogenic diseases & 0.53 & $0.25,1.14$ & 0.105 & 0.52 & $0.24,1.14$ & 0.103 \\
\hline Antiplatelet/ anticoagulation & 0.76 & $0.46,1.27$ & 0.300 & 0.71 & $0.41,1.23$ & 0.225 \\
\hline
\end{tabular}

*, $\mathrm{P}$ values were calculated after adjusting for age, sex, body mass index, current smoking, ApoE $\varepsilon 4$ carriers, ApoE $\varepsilon 2$ carriers, BP categories, diabetes, hyperlipidemia, cardiogenic diseases, and antiplatelet or anticoagulation medication. BP categories: (I) normal BP, $\mathrm{SBP}<120 \mathrm{mmHg}$ and DBP $<80 \mathrm{mmHg}$; (II) elevated systolic BP, SBP of 120 to $129 \mathrm{mmHg}$ and DBP $<80 \mathrm{mmHg}$; (III) stage 1 hypertension, SBP of 130 to $139 \mathrm{mmHg}$ or DBP of 80 to $89 \mathrm{mmHg}$; (IV) stage 2 hypertension, SBP $\geq 140 \mathrm{mmHg}$ or DBP $\geq 90 \mathrm{mmHg}$. BP, blood pressure; SBP, systolic blood pressure; DBP, diastolic blood pressure; IRR, incident rate ratio; Cl, confidence interval.
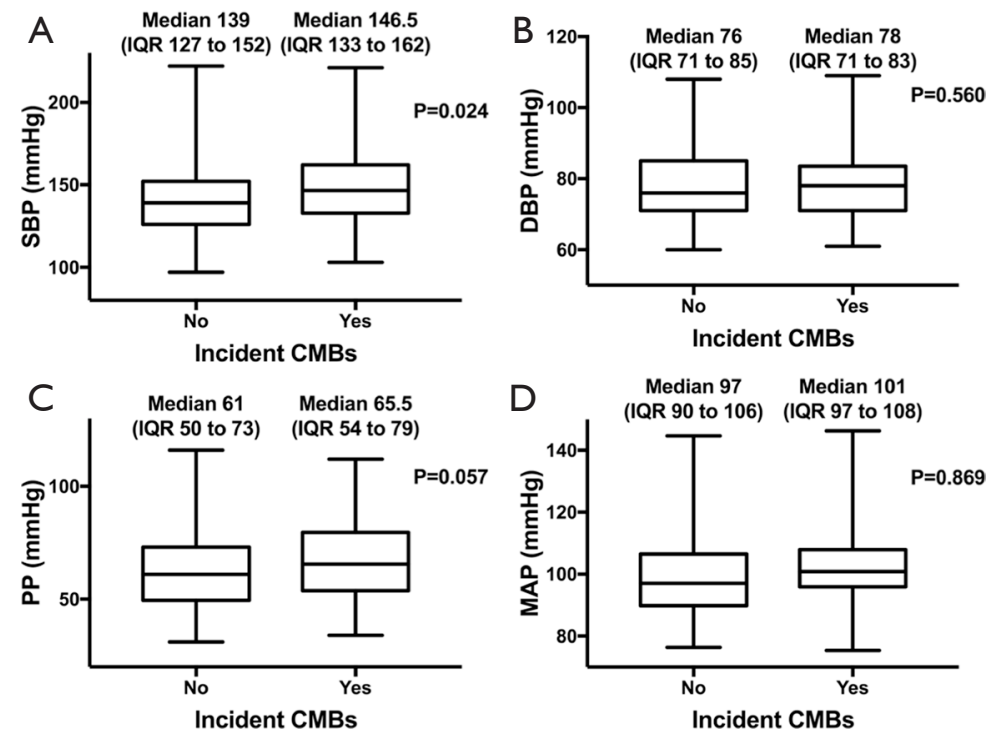

Figure 3 Baseline BP in participants with and without incident CMBs. Box and whisker plots show baseline BP in participants with no incident CMBs ( $\mathrm{n}=113)$ and with incident CMBs $(\mathrm{n}=46)$. Boxes show 25-75th percentile of BP, lines show median, and whiskers show range of BP. CMBs, cerebral microbleeds; BP, blood pressure; SBP, systolic blood pressure; DBP, diastolic blood pressure; PP, pulse pressure; MAP, mean arterial pressure; IQR, interquartile range 
2 hypertension defined by the 2017 ACC/AHA guidelines were approximately 3 times more likely to develop incident CMBs compared with normal BP in this elderly healthy population.

Hypertension defined as BP over 140/90 $\mathrm{mmHg}$ was considered a crucial risk factor of CMBs in the general population. In the middle-aged and elderly individuals in Rotterdam Scan Study (5), hypertension was associated with the presence of CMBs. One Swedish study found that hypertension was the major risk factor for $\mathrm{CMBs}$ in the general population (6). Longitudinal community studies $(7,8)$ also demonstrated that baseline hypertension was associated with development of new CMBs.

However, studies on the relationship between hypertension defined by the ACC/AHA guideline and CMBs are lacking. One cross-sectional study in routine health checkups (17) found that middle-aged participants with stage 1 hypertension had a higher prevalence of CMBs than those with normal BP, suggesting the clinical significance of this new definition of hypertension in predicting CMBs. Since cross-sectional studies proved only the association but not the causality, longitudinal studies were needed. Our 7 -year longitudinal study in the elderly population indicated that participants with baseline stage 1 hypertension were significantly more likely to have incident CMBs, especially numerous and strictly deep CMBs. This implied that a new, stricter BP control might help prevent CMBs.

The SPRINT Memory and Cognition IN Decreased Hypertension (SPRINT MIND) study (26) found intensive BP lowering to a SBP target $<120 \mathrm{mmHg}$ slowed the development of white matter lesions (another marker of CSVD). In our study, we found no association between the group with slightly elevated SBP (120 to $129 \mathrm{mmHg}$ ) and a higher incidence rate of CMBs.

Consistent with previous longitudinal studies $(7,8)$, we found that higher SBP rather than DBP at baseline was associated with incident $\mathrm{CMBs}$, suggesting that an aggressive anti-hypertensive treatment with a strict long-term control of SBP under $130 \mathrm{mmHg}$ might alleviate the progression of CMBs in the general population. This needs to be validated in randomized clinical trials.

A previous study found that cerebral small vessels were vulnerable to a wide pulse pressure (PP) because elevated PP could promote severe vascular dysfunction and lead to disruption of the neurovascular unit (27). Another study pointed out that aortic stiffness resulted in consequential increases in the amplitude of SBP and amplification of PP in the elderly population $(28,29)$, and eventually led to thinned-walled distal portion and cerebromicrovascular injury, an important contributing factor to the pathogenesis of CMBs $(30,31)$. Our results supported the associations between elevated SBP, PP and incident CMBs in an elderly population over 60 years of age.

ApoE gene encodes a protein involved in lipid and cholesterol metabolism and plays an important role in $\beta$-amyloid (A $\beta$ ) deposition and clearance (32). Pathology studies suggested that the $\varepsilon 2$ allele contributed to vascular ruptures and the $\varepsilon 4$ allele either contributed to increased $A \beta$ deposition or decreased $A \beta$ clearance in the vessels (33). Previous studies demonstrated that APOE $\varepsilon 4$ allele carriers possessed more prevalence of CMBs in the communitydwelling population $(5,34)$, while the associations between APOE $\varepsilon 2$ allele and CMBs remain uncertain (5,35-37). In this longitudinal study, we found APOE $\varepsilon 4$ associated with incident CMBs. No association was found between APOE $\varepsilon 2$ and incident CMBs.

The major strengths of this study are its populationbased design, long follow-up duration up to 7 years, and repeated imaging. We collected detailed and comprehensive characteristics of participants that enabled us to examine a variety of risk factors and to adjust for potential confounders. Our study has several limitations. First, T2*GRE sequence was applied for detecting CMBs at baseline, while SWAN was applied at follow-up. Theoretically, more CMBs could be detected on SWAN sequence compared to T2*-GRE, which might lead to an overestimation of the incidence rate of CMBs. However, since all participants underwent the same MRI sequence at the same time point, the variation of MRI sequence could be considered as a systematic error with limited impact when examining the associations between BP and incident CMBs. Second, BP was measured in a single day in the office. With regard to white coat effect, this might lead to misclassification. However, previous epidemiological studies revealed that BP measurements at a single visit were adequate for determining hypertensive status (38). Moreover, since $\mathrm{BP}$ was measured under the same circumstances for all the participants at baseline, the misclassification of BP categories due to white coat effect could be regarded as a limited systematic error. Third, we did not observe any impact of the BP control at follow-up on the incidence rate of CMBs (Table S4). This result needed to be further reexamined in the future community studies with repeated BP measurements throughout the follow-up period, since BP data at follow-up could not fully determine the status of BP control throughout the 7 years. Finally, there was an 
attrition bias during the follow-up period. People included in the analysis were younger and healthier than those lost to follow-up, leading to a probable bias in analyzing risk factors. However, even in this relatively healthy cohort, we found stage 1 hypertension associated with incident CMBs.

\section{Conclusions}

In conclusion, participants with baseline stage 1 and stage 2 hypertension had a significantly higher risk of incident CMBs in this 7-year longitudinal community cohort.

\section{Acknowledgments}

Funding: This study is funded by the National Key R\&D Program of China (2016YFC1300503, 2017YFC1308201), the National Natural Science Foundation of China (81971123), the Shanghai Municipal Science and Technology Major Project (No.2018SHZDZX01) and ZJLab, and the Research Center on Aging and Medicine, Fudan University.

\section{Footnotes}

Reporting Checklist: The authors have completed the STROBE reporting checklist. Available at http://dx.doi. org/10.21037/atm-20-5142

Data Sharing Statement: Available at http://dx.doi. org/10.21037/atm-20-5142

Peer Review File: Available at http://dx.doi.org/10.21037/ atm-20-5142

Conflicts of Interest: All authors have completed the ICMJE uniform disclosure form (available at http:// dx.doi.org/10.21037/atm-20-5142). All authors report grants from the National Key R\&D Program of China (2016YFC1300503, 2017YFC1308201), the National Natural Science Foundation of China (81971123), the Shanghai Municipal Science and Technology Major Project (No.2018SHZDZX03) and Zhangjiang Lab, and the Research Center on Aging and Medicine, Fudan University, during the conduct of the study. The authors have no other conflicts of interest to declare.

Ethical Statements: The authors are accountable for all aspects of the work in ensuring that questions related to the accuracy or integrity of any part of the work are appropriately investigated and resolved. This study was approved by the medical ethics committee of Huashan Hospital, Fudan University, Shanghai, China (ID: 2016359), and conducted in accordance with Chinese Good Clinical Practice of Pharmaceutical Products and the Declaration of Helsinki (as revised in 2013). Written informed consent was obtained from all participants or their legally acceptable representative.

Open Access Statement: This is an Open Access article distributed in accordance with the Creative Commons Attribution-NonCommercial-NoDerivs 4.0 International License (CC BY-NC-ND 4.0), which permits the noncommercial replication and distribution of the article with the strict proviso that no changes or edits are made and the original work is properly cited (including links to both the formal publication through the relevant DOI and the license). See: https://creativecommons.org/licenses/by-nc-nd/4.0/.

\section{References}

1. Wardlaw JM, Smith EE, Biessels GJ, et al. Neuroimaging standards for research into small vessel disease and its contribution to ageing and neurodegeneration. Lancet Neurol 2013;12:822-38.

2. Charidimou A, Kakar P, Fox Z, et al. Cerebral microbleeds and recurrent stroke risk: systematic review and metaanalysis of prospective ischemic stroke and transient ischemic attack cohorts. Stroke 2013;44:995-1001.

3. Martinez-Ramirez S, Greenberg SM, Viswanathan A. Cerebral microbleeds: overview and implications in cognitive impairment. Alzheimers Res Ther 2014;6:33.

4. Altmann-Schneider I, Trompet S, de Craen AJ, et al. Cerebral microbleeds are predictive of mortality in the elderly. Stroke 2011;42:638-44.

5. Poels MM, Vernooij MW, Ikram MA, et al. Prevalence and risk factors of cerebral microbleeds: an update of the Rotterdam scan study. Stroke 2010;41:S103-6.

6. Elmståhl S, Ellstrom K, Siennicki-Lantz A, et al. Association between cerebral microbleeds and hypertension in the Swedish general population "Good Aging in Skane" study. J Clin Hypertens (Greenwich) 2019;21:1099-107.

7. Ding J, Sigurdsson S, Garcia M, et al. Risk Factors Associated With Incident Cerebral Microbleeds According to Location in Older People: The Age, Gene/Environment Susceptibility (AGES)-Reykjavik Study. JAMA Neurol 
2015;72:682-8.

8. Poels MM, Ikram MA, van der Lugt A, et al. Incidence of cerebral microbleeds in the general population: the Rotterdam Scan Study. Stroke 2011;42:656-61.

9. A Randomized Trial of Intensive versus Standard BloodPressure Control. N Engl J Med 2017;377:2506.

10. Whelton PK, Carey RM, Aronow WS, et al. 2017 ACC/ AHA/AAPA/ABC/ACPM/AGS/APhA/ASH/ASPC/ NMA/PCNA Guideline for the Prevention, Detection, Evaluation, and Management of High Blood Pressure in Adults: A Report of the American College of Cardiology/ American Heart Association Task Force on Clinical Practice Guidelines. Circulation 2018;138:e484-594.

11. Kitagawa K, Yamamoto Y, Arima H, et al. Effect of Standard vs Intensive Blood Pressure Control on the Risk of Recurrent Stroke: A Randomized Clinical Trial and Meta-analysis. JAMA Neurol 2019;76:1309-18.

12. SPS3 Study Group, Benavente OR, Coffey CS, et al. Bloodpressure targets in patients with recent lacunar stroke: the SPS3 randomised trial. Lancet 2013;382:507-15.

13. Bress AP, Kramer H, Khatib R, et al. Potential Deaths Averted and Serious Adverse Events Incurred From Adoption of the SPRINT (Systolic Blood Pressure Intervention Trial) Intensive Blood Pressure Regimen in the United States: Projections From NHANES (National Health and Nutrition Examination Survey). Circulation 2017;135:1617-28.

14. Schiffrin EL. New blood pressure cut-offs, prevalence of hypertension and control, and mood disorders: are patients benefitting from lower cut-offs for defining hypertension? Eur Heart J 2019;40:739-42.

15. Brunström M, Carlberg B. Association of Blood Pressure Lowering With Mortality and Cardiovascular Disease Across Blood Pressure Levels: A Systematic Review and Meta-analysis. JAMA Intern Med 2018;178:28-36.

16. Atasoy S, Johar H, Peters A, et al. Association of hypertension cut-off values with 10 -year cardiovascular mortality and clinical consequences: a real-world perspective from the prospective MONICA/KORA study. Eur Heart J 2019;40:732-8.

17. Nam KW, Kwon HM, Jeong HY, et al. Cerebral Small Vessel Disease and Stage 1 Hypertension Defined by the 2017 American College of Cardiology/American Heart Association Guidelines. Hypertension 2019;73:1210-6.

18. Ding D, Zhao Q, Guo Q, et al. The Shanghai Aging Study: study design, baseline characteristics, and prevalence of dementia. Neuroepidemiology 2014;43:114-22.

19. Xia Y, Shen Y, Wang Y, et al. White matter hyperintensities associated with progression of cerebral small vessel disease: a 7-year Chinese urban community study. Aging (Albany NY) 2020;12:8506-22.

20. Mok V, Srikanth V, Xiong Y, et al. Race-ethnicity and cerebral small vessel disease--comparison between Chinese and White populations. Int J Stroke. 2014;9 Suppl A100:36-42.

21. Greenberg SM, Vernooij MW, Cordonnier C, et al. Cerebral microbleeds: a guide to detection and interpretation. Lancet Neurol 2009;8:165-74.

22. Gregoire SM, Chaudhary UJ, Brown MM, et al. The Microbleed Anatomical Rating Scale (MARS): reliability of a tool to map brain microbleeds. Neurology 2009;73:1759-66.

23. Wu S, Huang Z, Yang X, et al. Cardiovascular events in a prehypertensive Chinese population: four-year follow-up study. Int J Cardiol 2013;167:2196-9.

24. Vernooij MW, van der Lugt A, Ikram MA, et al. Prevalence and risk factors of cerebral microbleeds: the Rotterdam Scan Study. Neurology 2008;70:1208-14.

25. Loehrer E, Ikram MA, Akoudad S, et al. Apolipoprotein E genotype influences spatial distribution of cerebral microbleeds. Neurobiol Aging 2014;35:899-905.

26. Kjeldsen SE, Narkiewicz K, Burnier M, et al. Intensive blood pressure lowering prevents mild cognitive impairment and possible dementia and slows development of white matter lesions in brain: the SPRINT Memory and Cognition IN Decreased Hypertension (SPRINT MIND) study. Blood Press 2018;27:247-8.

27. de Montgolfier O, Pincon A, Pouliot P, et al. High Systolic Blood Pressure Induces Cerebral Microvascular Endothelial Dysfunction, Neurovascular Unit Damage, and Cognitive Decline in Mice. Hypertension 2019;73:217-28.

28. Diaz-Otero JM, Garver H, Fink GD, et al. Aging is associated with changes to the biomechanical properties of the posterior cerebral artery and parenchymal arterioles. Am J Physiol Heart Circ Physiol 2016;310:H365-75.

29. Phan TS, Li JK, Segers P, et al. Misinterpretation of the Determinants of Elevated Forward Wave Amplitude Inflates the Role of the Proximal Aorta. J Am Heart Assoc 2016;5:e003069.

30. O'Rourke MF, Safar ME. Relationship between aortic stiffening and microvascular disease in brain and kidney: cause and logic of therapy. Hypertension 2005;46:200-4.

31. Sörös P, Whitehead S, Spence JD, et al. Antihypertensive treatment can prevent stroke and cognitive decline. Nat Rev Neurol 2013;9:174-8. 
32. Bu G. Apolipoprotein E and its receptors in Alzheimer's disease: pathways, pathogenesis and therapy. Nat Rev Neurosci 2009;10:333-44.

33. Auriel E, Greenberg SM. The pathophysiology and clinical presentation of cerebral amyloid angiopathy. Curr Atheroscler Rep 2012;14:343-50.

34. Sveinbjornsdottir S, Sigurdsson S, Aspelund T, et al. Cerebral microbleeds in the population based AGESReykjavik study: prevalence and location. J Neurol Neurosurg Psychiatry 2008;79:1002-6.

35. Groot C, Sudre CH, Barkhof F, et al. Clinical phenotype, atrophy, and small vessel disease in APOEepsilon2 carriers

Cite this article as: Xia Y, Wang Y, Yang L, Wang Y, Liang X, Zhao Q, Wu J, Chu S, Liang Z, Ding H, Ding D, Cheng X, Dong Q. Incident cerebral microbleeds and hypertension defined by the 2017 ACC/AHA Guidelines. Ann Transl Med 2021;9(4):314. doi: 10.21037/atm-20-5142 with Alzheimer disease. Neurology 2018;91:e1851-9.

36. Kim M, Bae HJ, Lee J, et al. APOE epsilon2/epsilon4 polymorphism and cerebral microbleeds on gradient-echo MRI. Neurology 2005;65:1474-5.

37. Lemmens R, Gorner A, Schrooten M, et al. Association of apolipoprotein $\mathrm{E}$ epsilon2 with white matter disease but not with microbleeds. Stroke 2007;38:1185-8.

38. Markovic N, Olomu IN, Bunker CH, et al. Adequacy of a single visit for classification of hypertensive status in a Nigerian civil servant population. Int J Epidemiol 1994;23:723-9. 


\section{Supplementary}

Table S1 Characteristics of the study population at baseline and follow-up ( $\mathrm{n}=159)$

\begin{tabular}{|c|c|c|c|c|}
\hline Characteristics & Baseline $(n=159)$ & Follow-up ( $\mathrm{n}=159)$ & Change & $P$ value \\
\hline Age, y, median (IQR) & 67 (62.7 to 72.2$)$ & 73.9 (69.0 to 78.4$)$ & 6.8 (6.7 to 7.3$)$ & - \\
\hline Sex, male, n (\%) & $71(44.7)$ & $71(44.7)$ & - & - \\
\hline Education, y, median (IQR) & $12(9$ to 15$)$ & $12(9$ to 15$)$ & - & - \\
\hline Body mass index, $\mathrm{kg} / \mathrm{m}^{2}$, median (IQR) & 24.5 (21.9 to 27.3$)$ & 23.4 (21.6 to 26.1$)$ & $-1.2(-2.1$ to 0.3$)$ & 0.023 \\
\hline Current smoking, $\mathrm{n}(\%)$ & $17(10.7)$ & $11(6.9)$ & $-6(3.8)$ & 0.235 \\
\hline ApoE $\varepsilon 4$ carriers, $n(\%)$ & $23(14.8)$ & $23(14.8)$ & - & - \\
\hline ApoE $\varepsilon 2$ carriers, n (\%) & $26(16.8)$ & $26(16.8)$ & - & - \\
\hline Hyperlipidemia, n (\%) & $61(38.4)$ & $69(43.4)$ & $8(5.0)$ & 0.361 \\
\hline Cardiogenic diseases, n (\%) & $17(10.7)$ & $24(15.1)$ & $7(4.4)$ & 0.241 \\
\hline \multicolumn{5}{|l|}{ Medication use, n (\%) } \\
\hline Antihypertensive & $73(45.9)$ & $90(56.6)$ & $17(10.7)$ & 0.056 \\
\hline Antidiabetic & $16(10.1)$ & $30(18.9)$ & $14(8.8)$ & 0.026 \\
\hline Lipid lowering & $6(3.8)$ & $30(18.9)$ & $24(15.1)$ & $<0.001$ \\
\hline Antiplatelet/anticoagulation & $29(18.2)$ & $25(15.7)$ & $-4(2.5)$ & 0.550 \\
\hline \multicolumn{5}{|l|}{ Prevalence of microbleeds, n (\%) } \\
\hline Lobar or infratentorial microbleeds & $10(6.3)$ & $39(24.5)$ & $29(18.2)$ & $<0.001$ \\
\hline
\end{tabular}

$I Q R$, interquartile range. 
Table S2 Baseline characteristics of all individuals $(\mathrm{n}=317)$

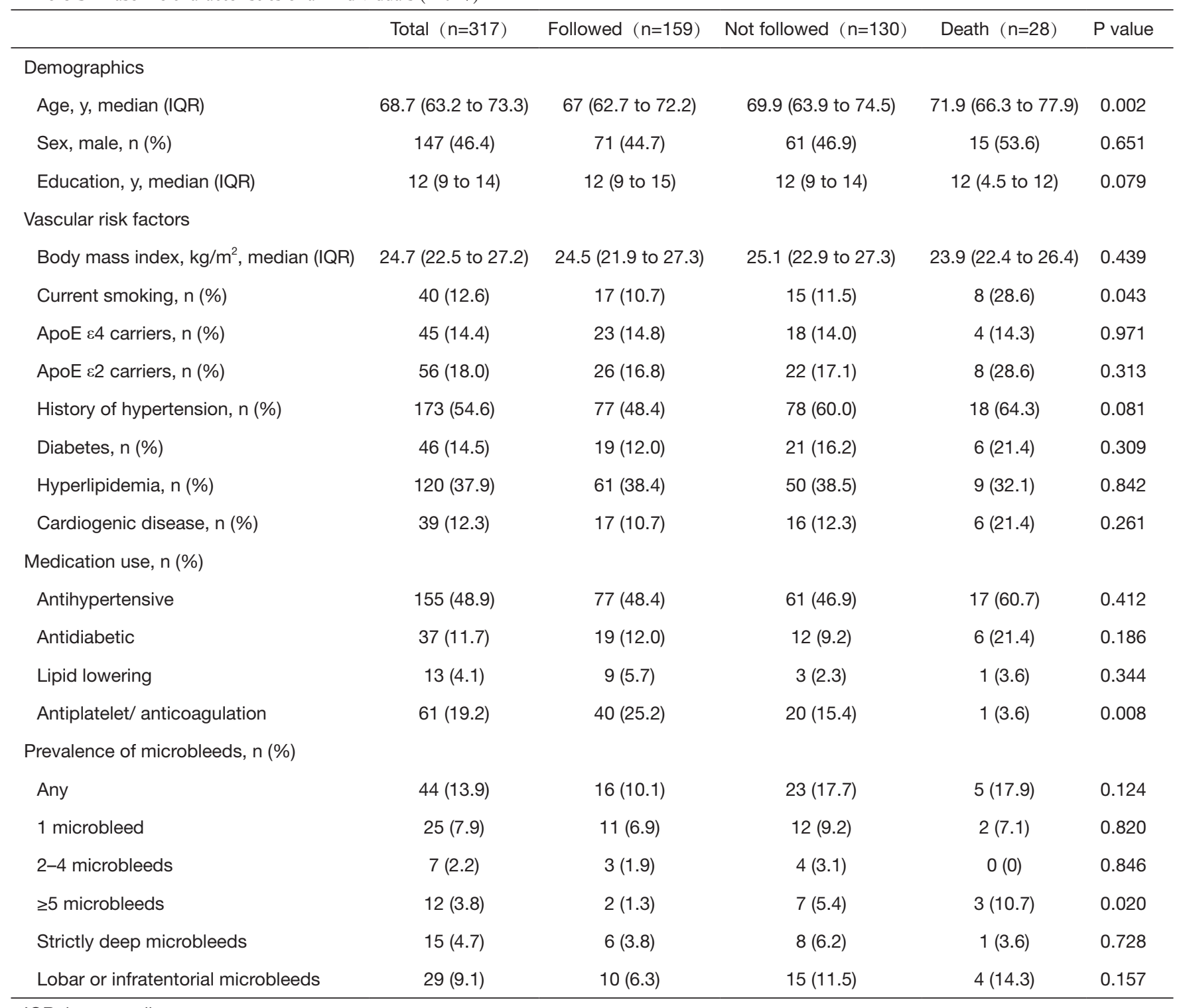

$I Q R$, interquartile range. 
Table S3 No. (Percentage) of participants at baseline and follow-up with cerebral microbleeds in four BP groups

\begin{tabular}{|c|c|c|c|c|c|c|}
\hline & Total & Normal BP & Elevated SBP & Stage 1 hypertension & Stage 2 hypertension & $P$ value \\
\hline Any microbleeds & $16(10.1)$ & $1(4.8)$ & $1(6.7)$ & $4(11.4)$ & $10(11.4)$ & 0.906 \\
\hline 1 microbleed & $11(6.9)$ & $0(0)$ & $1(6.7)$ & $3(8.6)$ & $7(8.0)$ & 0.650 \\
\hline 2-4 microbleeds & $3(1.9)$ & $1(4.8)$ & $0(0)$ & $1(2.9)$ & $1(1.1)$ & 0.507 \\
\hline Strictly deep microbleeds & $6(3.8)$ & $1(4.8)$ & $0(0)$ & $1(2.9)$ & $4(4.5)$ & 1.000 \\
\hline Lobar or infratentorial microbleeds & $10(6.3)$ & $0(0)$ & $1(6.7)$ & $3(8.6)$ & $6(6.8)$ & 0.663 \\
\hline \multicolumn{7}{|c|}{ No. (Percentage) of participants at follow-up } \\
\hline Any microbleeds & $53(33.3)$ & $4(19.0)$ & $4(26.7)$ & $11(31.4)$ & $34(38.6)$ & 0.354 \\
\hline$\geq 5$ microbleeds & $6(3.8)$ & $0(0)$ & $0(0)$ & $2(5.7)$ & $4(4.5)$ & 0.795 \\
\hline Strictly deep microbleeds & $14(8.8)$ & $0(0)$ & $0(0)$ & $3(8.6)$ & $11(12.5)$ & 0.226 \\
\hline Lobar or infratentorial microbleeds & $39(24.5)$ & $4(19.0)$ & $4(26.7)$ & $8(22.9)$ & $23(26.1)$ & 0.924 \\
\hline
\end{tabular}

BP, blood pressure; SBP, systolic blood pressure. 
Table S4 Univariable and multivariable analyses between possible predictors and incident cerebral microbleeds

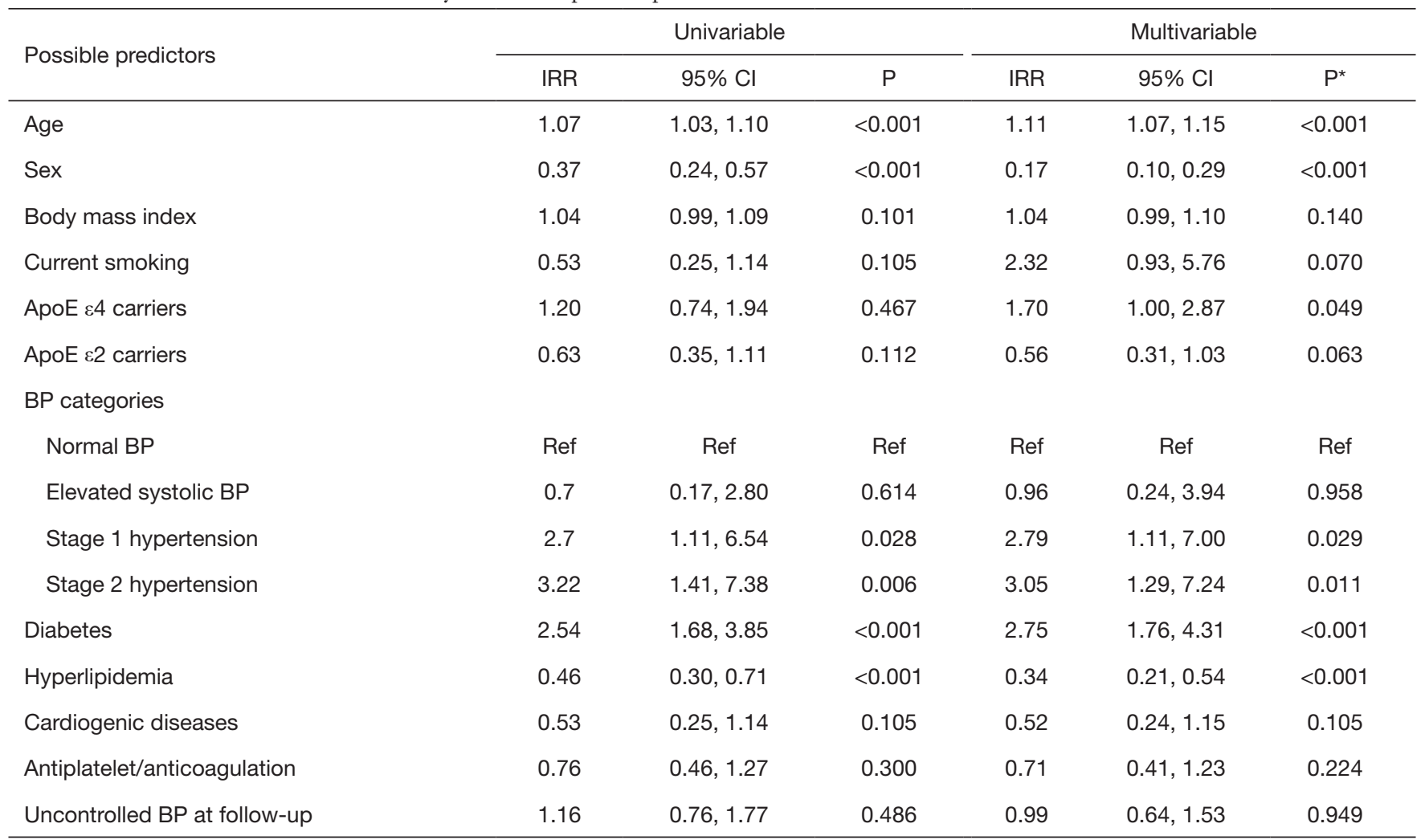

*, P values were calculated after adjusting for age, sex, body mass index, current smoking, ApoE $\varepsilon 4$ carriers, ApoE $\varepsilon 2$ carriers, BP categories, diabetes, hyperlipidemia, cardiogenic diseases, antiplatelet or anticoagulation medication and uncontrolled BP at follow-up. BP categories: (I) normal BP, SBP $<120 \mathrm{mmHg}$ and DBP $<80 \mathrm{mmHg}$; (II) elevated systolic BP, SBP of 120 to $129 \mathrm{mmHg}$ and DBP <80 mmHg; (III) stage 1 hypertension, SBP of 130 to $139 \mathrm{mmHg}$ or DBP of 80 to $89 \mathrm{mmHg}$; (IV) stage 2 hypertension, SBP $\geq 140 \mathrm{mmHg}$ or $\mathrm{DBP} \geq 90 \mathrm{mmHg}$. Uncontrolled BP at follow-up: SBP $\geq 130 \mathrm{mmHg}$ or DBP $\geq 80 \mathrm{mmHg}$. BP, blood pressure; SBP, systolic blood pressure; $\mathrm{DBP}$, diastolic blood pressure; IRR, incident rate ratio. 